\title{
OPTICAL SPECTROSCOPY OF HIGHLY IONIZED NEON
}

\author{
L.J. LEMBO, Ch. STOLLER, K. DANZMANN, W.E. MEYERHOF and T.W. HANSCH* \\ Department of Physics, Stanford University. CA 94305. USA
}

\section{R. GERSON}

Hamamatusu Corporation, Middlesex, NJ 08846, USA

\begin{abstract}
Optical spectroscopy studies have been performed on slow, highly charged neon recoil ions. Rydberg states were populated by electron capture from sodium. These spectra reveal the influence of core interactions on the alignment of states populated by electron capture processes.
\end{abstract}

The recent development of sources such as the recoil ion source [1], producing highly charged, low energy (HQLE) ions presents the possibility of bringing highresolution laser spectroscopy to the study of highly charged lons. A prerequisite for performing these measurements is the preparation of the HQLE ions quantum states which participate in transitions accessible to tunable lasers. For this purpose, one can exploit electron transfer collisions which selectively populate highly excited states of slow ions. Optical studies on these ions would also permit a thorough understanding of the electron capture process itself.

Knowledge of the partial cross sections for capture into the various $(n, l, m)$ substates of the projectile ion plays an important role in these endeavors. Theories of varying complexity exist for predicting these distributions. The well-known classical barrier model (CBM) is a simple yet useful model for describing electron transfer [2]. It allows a prediction of the principal (n) quantum number characterizing the states most likely to be populated in collisions involving slow, highly ionized species. More intricate theories are necessary for predicting the distribution of angular momentum $(l)$ and magnetic $(m)$ substates populated by electron transfer. Such distributions have been obtained for collision systems consisting of bare projectile ions and $H(1 s)$ atoms [3]. Calculation of the $l, m$ distributions demands that one take proper account of the Stark mixing of projectile ion substates in the electric field of the residual target ion. When projectle ions with core electrons are used, the $l$ substates into which electrons are captured become nondegenerate. One must therefore reconsider

* This work is supported in part by the National Science Foundation, under Grants PHY-83-13676 and PHY-8308271 . the effect of Stark mixing. This is an open question for both the experimental and the theoretical points of view.

One salient feature of these capture processes is the unequal population of $m$ substates (alignment). This manifests itself experimentally as a nonzero polarization of the spontancous emission radiated subsequent to capture [4]. Using a grating spectrometer, we have performed optical spectroscopic studies in the visible and near UV, which resolve the fine structure of the transitions. Together with our ongoing polarization studies, these spectra may provide evidence for the effect of core interactions on the $l, m$ distribution populated by the primary capture process and changed by postcollision Stark mixing.

We determined wavelengths for $\Delta n=-1$ transitions following electron capture from atomic sodium into Rydberg states of slow, highly charged neon ions. Charge-state selected $\mathrm{Ne}^{* *}$ recoll ions, provided by the Stanford recoil ion souce [5], are focussed onto an atomic Na vapor target, produced by an effusive alkali beam oven maintained at a temperature of approximately $400^{\circ} \mathrm{C}$. Recoil ion charge states with $q=10$ to $q=6$ with energies of $0.5 q \mathrm{keV}$ were extracted. We used typical recoil ion currents of up to $10 \mathrm{pA}$ for bare neon and up to $300 \mathrm{pA}$ for $\mathrm{Ne}^{x}$ and lower charge states. Photons emerging from the $\mathrm{Ne}^{4 !}+\mathrm{Na}$ interaction region are focussed onto the slit of a Jobin Yvon HR-320 normal incidence grating spectrometer wh $0.32 \mathrm{~m}$ focal length. The instrument is equipped with a $24001 / \mathrm{mm}$ holographic grating and has a useful spectral response from 250 to $600 \mu \mathrm{m}$. The photocathode of a Hamamatsu photon-counting image acquisition system (PIAS) is positioned in the spectrometer focal plane. Two dimensional images of the focal plane are acquired and processed into wavelength spectra. A spectral range of 
$18 \mathrm{~nm}$ can be covered in one exposure. Typical data acquistion times range from $10 \mathrm{~min}$ to $2 \mathrm{~h}$. The combination of PIAS spatial resolution, spectrometer focal plane dispersion and spectrometer slit width gave a spectral resolution of $50 \mathrm{pm}$.

All spectra show evidence for photons emitted in transitions following electron capture into states whose principal quantum number is given by the CBM. For ions with initial $q \geqq 8$ the measured wavelengths have values agreeing with the corresponding hydrogen-like predictions. The agrement of experimental hydrogenlike wavelengths in these cases as well as the absence of any structure in the spectral lines, show that the interaction of the captured electron of the $\mathrm{Ne}^{\mathrm{x}+*}$ and $\mathrm{Ne}^{7 * *}$ ions is undetectable with our present spectral resolution fig. 1). Using the Hartree-Fock code RCN/RCG [6]. we have calculated the level structure of the systems under investigation. We find perfect agreement with our measurements, if we assume that all the detected photons are emitted from ion states having ground state
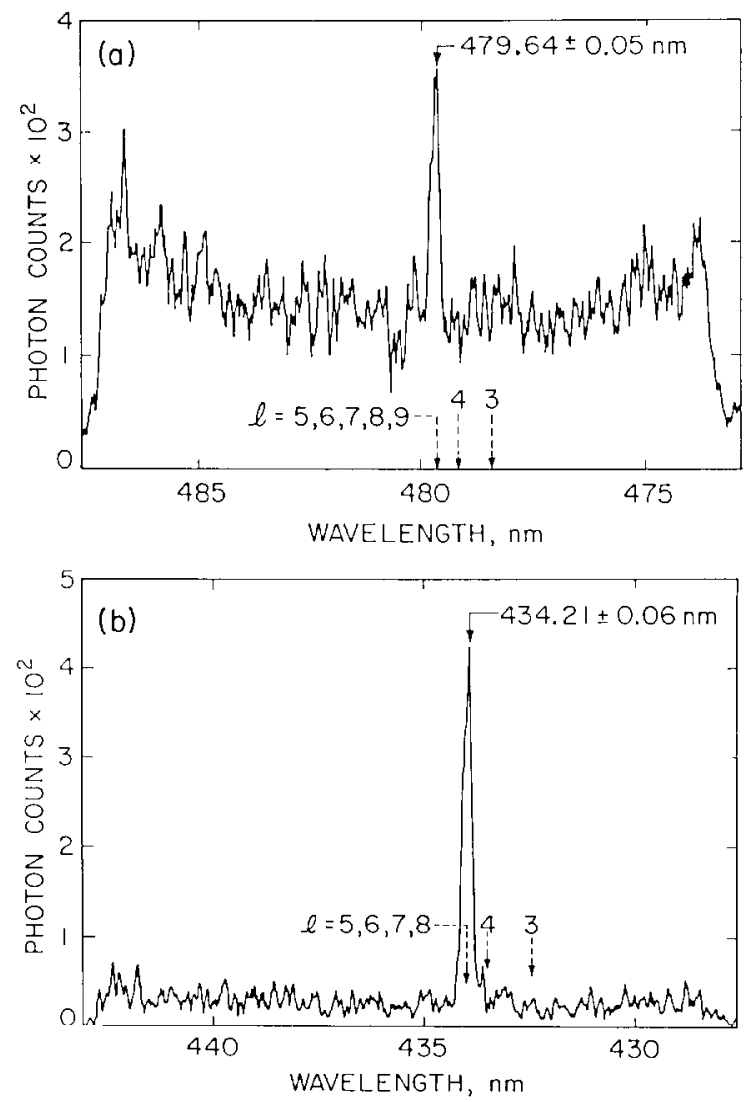

Fig. 1. Spectrum for a $n=9$ to 8 transition of $\mathrm{Ne}^{1+*}$ following capture into $0.5 q \mathrm{keV} \mathrm{Ne}{ }^{x}$. showing an unresolved line at the hydrogen-like wavelength. Hartree-Fock predictions for transitions originating at various angular momentum (/) sublevels are indicated by broken lines. Ground-state cores are assumed corc configurations and orbital angular momentum quantum numbers $l \geqq 5$. Metastable cores would interact with the outer (captured) electron strongly enough to give rise to resolvable fine structure.

In contrast to the $\mathrm{Ne}^{y+}+\mathrm{Na}$ and $\mathrm{Ne}^{x+}+\mathrm{Na}$ cases, structure is evident in the spectra for transitions following electron capture into projectile ions having $\mathrm{L}$-shell $(n=2)$ electrons in their ground-state cores. The $n=8$ to $n=7$ transition following capture into $\mathrm{Ne}^{7}$ has a hydrogen-like wavelength of $\lambda=3888 \AA$. The $n=7$ to $n=6$ transition following capture into $\mathrm{Ne}^{6+}$ has a hydrogen-like wavelength of $\lambda=3435 \AA$; the observed spectrum is shown in fig. 2. We believe the observed structure to be caused by degeneracy splitting of various I sublevels via the interaction with core electrons. But for these cases we find only qualitative agreement between the predictions of the Hartree-Fock calculations, including configuration interactions and the experimental spectra. Work is in progress to identify the various spectral features of these transitions and to explore the influence of excited core configurations and spectator electrons.
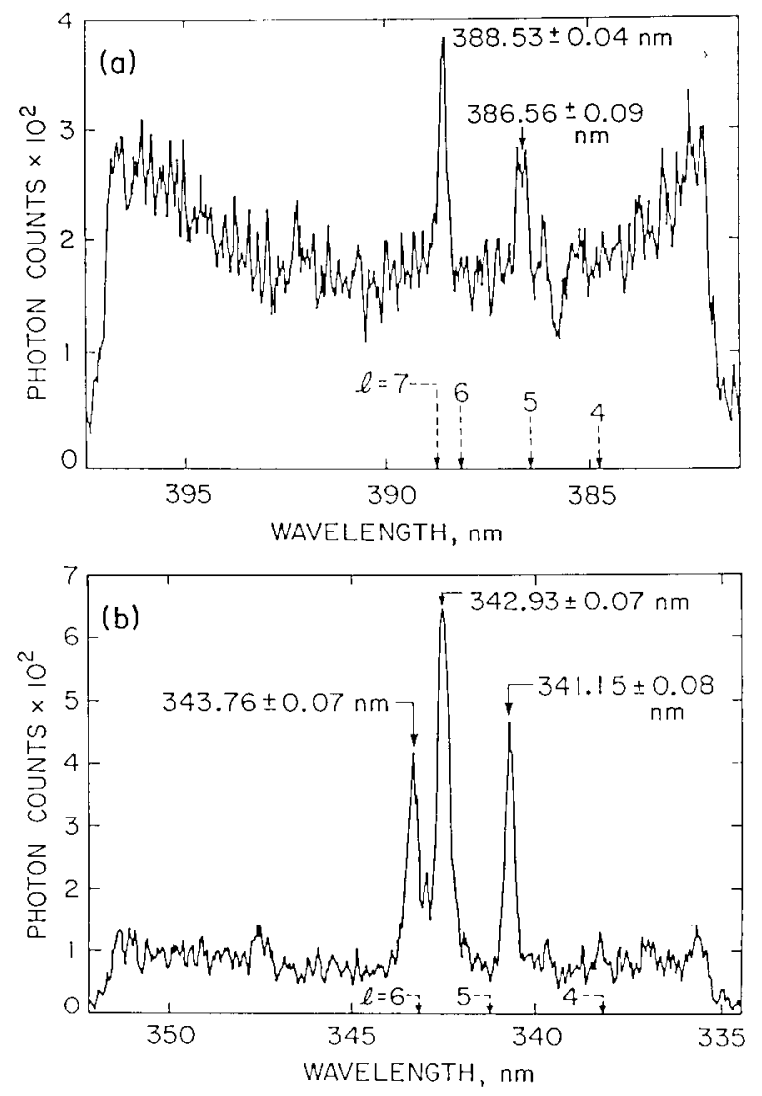

Fig. 2. Spectrum for an $n=7$ to 6 transition of $\mathrm{Ne}^{5+*}$ following capture into $0.5 q \mathrm{keV} \mathrm{Ne}{ }^{\mathrm{f}}$. Hartree-Fock predictions indicated as in fig. 1. 
Table 1

Summary of results

$\mathrm{Ne}^{q+}+\mathrm{Na} \rightarrow \mathrm{Ne}^{(q)}(1)+*(n)+\mathrm{Na}{ }^{+}$

\begin{tabular}{|c|c|c|c|c|c|c|c|}
\hline 4 & $n_{\mathrm{CBM}}$ & $\begin{array}{l}\text { Transi- } \\
\text { tion }\end{array}$ & $\begin{array}{l}\text { Rydberg- } \\
\text { like } \lambda \\
{[\mathrm{nm}]}\end{array}$ & $\begin{array}{l}\text { Mcasured } \\
\lambda \\
{[\mathrm{nm}]}\end{array}$ & $\begin{array}{l}\text { Relative } \\
\text { intensity }\end{array}$ & $\begin{array}{l}P_{\text {theory }} \\
\text { (Polarization) }\end{array}$ & $P_{\text {exp }}$ \\
\hline 10 & 10 & $10 \rightarrow 9$ & 388.5 & $388.5-0.15$ & & 0.32 & $0.32 \pm 0.04$ \\
\hline 9 & 10 & $\begin{aligned} 10 & \rightarrow 9 \\
9 & \rightarrow 8\end{aligned}$ & $\begin{array}{l}479.62 \\
343.07\end{array}$ & $\begin{array}{l}479.64 \pm 0.05 \\
343.07 \pm 0.07\end{array}$ & $\begin{array}{l}1 \\
1.8\end{array}$ & $\begin{array}{l}0.31 \\
0.31\end{array}$ & $\begin{array}{l}0.31 \pm 0.02 \\
0.23 \pm 0.03\end{array}$ \\
\hline 8 & 9 & $\begin{aligned} 10 & \rightarrow 9 \\
9 & \rightarrow 8 \\
8 & \rightarrow 7\end{aligned}$ & $\begin{array}{l}607.0 \\
434.19 \\
297.68\end{array}$ & $\begin{array}{l}\text { Absent } \\
434.21 \pm 0.06 \\
297.68+0.04\end{array}$ & $\begin{array}{c}<0.22 \\
1 \\
1.1\end{array}$ & $\begin{array}{l}- \\
0.30 \\
0.30\end{array}$ & $\begin{array}{l}0.30 \pm 0.01 \\
0.23+0.04\end{array}$ \\
\hline 7 & 8 & $8 \rightarrow 7$ & 388.81 & $\begin{array}{l}386.54 \pm 0.06 \\
388 \pm 0.04\end{array}$ & $\begin{array}{l}0.45 \\
1\end{array}$ & 0.30 & $0.23 \pm 0.03$ \\
\hline 6 & 7 & $\begin{array}{l}8 \rightarrow 7 \\
7 \rightarrow 6\end{array}$ & $\begin{array}{l}529.2 \\
343.48\end{array}$ & $\begin{array}{l}\text { Absent } \\
343.70 \pm 0.07 \\
342.93 \pm 0.07 \\
341.15+0.08\end{array}$ & $\begin{array}{c}<0.1 \\
0.50 \\
1 \\
0.45\end{array}$ & 0.28 & $0.11+0.02$ \\
\hline 5 & 6 & $\begin{array}{l}7 \rightarrow 6 \\
6 \rightarrow 5\end{array}$ & $\begin{array}{l}494.6 \\
298.23\end{array}$ & $\begin{array}{l}\text { Absent } \\
297.68 \pm 0.08 \\
296.80 \pm 0.08 \\
296.28 \pm 0.08\end{array}$ & $\begin{array}{c}<1.2 \\
0.4 \\
1 \\
0.4\end{array}$ & 0.28 & $0.07 \pm 0.02$ \\
\hline
\end{tabular}

We have measured the polarization of the observed spectral lines emitted at right angles to the direction of the recoil ion beam. For these measurements we used an KCA 8850 photomultiplier and restricted the spectral range by the insertion of narrowband interference filters. For transitions showing fine-structure splitting only the average polarization of the fine-structure components could thus be determined.

We define the polarization $P$ in terms of the radiation polarized parallel and perpendicular to the ion path ( $I_{\|}$and $I_{\perp}$, respectively), as

$P=\left(I_{\|}-I_{\perp}\right) /\left(I_{\|}+I_{\perp}\right)$

In our preliminary polarization studies (see table 1) we have seen that radiation emitted in the transitions seen as structureless is highly polarized along the ion path. The degree of polarization can be explained by assuming the $(\mathrm{l} m)$ distributions calculated for bare projectiles and $\mathrm{H}(1 \mathrm{~s})$ targets [3], i.e. a very high population of $m=0, \pm 1$ substates.

On the other hand, radiation emitted in transitions whose spectra show structure is significantly depolarized with respect to those whose spectra are structureless. In these cases the relative population of $\mathrm{m}$ substates is more similar to a statistical distribution.

This suggests that the $(l, m)$ distribution after electron capture is affected by the degeneracy breakdown of the $l$ sublevels. A possible explanation is the inhibition of the linear Stark effect. Postcollision Stark mixing of the angular momentum substates (the magnetic quantum number being unaffected) could serve to increase the alignment of high angular momentum states. These states, in turn, dominate the light emission in the visible and near UV.

\section{References}

[1] C.L. Cocke, Phys. Rev. A20 (1979) 749.

[2] H. Ryufuku, K. Sasaki and T. Watanabe, Phys. Rev. A21 (1980) 745 .

[3] A. Salin, J. Phys. (Paris) 45 (1984) 671.

[4] L.J. Lembo, K. Danzmann, Ch. Stoller, W.E. Meyerhof and T.W. Hänsch, Phys. Rev. Lett. 55 (1985) 1874

[5] Ch. Stoller, L.J. Lembo, K. Danzmann and W.E. Mever hof. Proc. 14th ICPEAC (1985) p. 447.

[6] R.D. Cowan, The Theory of Atonic Structure and Spectra (University of California Press, 1981). 